\title{
Dapagliflozin in the treatment of patients with heart failure with reduced left ventricular ejection fraction - a practical approach
}

\author{
Jadwiga Nessler \\ Department of Coronary Disease and Heart Failure, Institute of Cardiology, Jagiellonian University Medical College, Krakow, Poland
}

Adv Interv Cardiol 2021; 17, 2 (64): 135-140

DOI: https://doi.org/10.5114/aic.2021.107491

\begin{abstract}
Because of its frequent occurrence, poor prognosis, low quality of life of patients accompanying the disease and high costs associated with treatment heart failure is a significant health problem in modern cardiology. The treatment of patients with heart failure and reduced ejection fraction (HFrEF) is supported by large-scale randomized clinical trials (RCTs), reflected in the ESC/HFA treatment recommendations. The use of $\beta$-blockers, inhibitors of the renin-angiotensin aldosterone system (RAA), including angiotensin converting enzyme ACE/ARB inhibitors, angiotensin and neprilysin receptor blockers (ARNI) and mineralocorticoid receptor antagonists (MRA) have been a solid basis for triple HFrEF therapy over the last few years, partially altering the natural history of patients with HF. Despite this, the 5-year survival rate in HF is still lower than for some types of cancer. Therefore, the search for new drugs to improve the prognosis of this disease is still ongoing. A new group of drugs - the so-called flozins, i.e. - is a great hope for a change in the natural history of heart failure. The following article presents practical aspects of using these drugs in the treatment of patients with HFrEF, taking into account both the inclusion criteria and various clinical profiles of patients.
\end{abstract}

Key words: heart failure with reduced left ventricular ejection fraction (HFrEF), flozins - sodium-glucose cotransporter type 2 (SGLT-2) inhibitors (SGLT-2), dapagliflozin, indications for use, patients' clinical profiles.

\section{Introduction}

Heart failure (HF) is the end-stage of many heart diseases, and its prevalence is increasing worldwide. Over the past dozen months or so, the COVID-19 pandemic has further worsened the situation of patients with HF. HF has become an invisible epidemic. Patients have stopped coming for follow-up appointments - mainly out of fear of being infected with the SARS-CoV2 coronavirus - or worse still, many have discontinued their prescribed therapy. This is why implementing a treatment with documented efficacy which does not require a complicated dosage regimen acquires such great importance nowadays. In recent years, there have been significant advances in the diagnosis and treatment of patients with HF with reduced left ventricular ejection fraction (HFrEF), resulting in reductions in cardiovascular (CV) and all-cause mortality, and a decrease in the rate of hospitalisation. The treatment of patients with HFrEF is supported by the findings of multi-centre randomised placebo-controlled clinical trials (RCTs), which are reflected in guidelines for the management of heart failure. Over the past years, $\beta$-blockers, inhibitors of the renin-angiotensin-aldosterone (RAA) system including angiotensin-converting enzyme inhibitors (ACEls), angiotensin receptor blockers (ARBs), combinations of ARBs and angiotensin receptor-neprilysin inhibitors (ARNIs), as well as mineralocorticoid receptor antagonists (MRAs), have provided a solid foundation for triple HFrEF therapy, partially changing the natural course of the disease in patients with HF. Despite this, however, the 5-year survival rate for $\mathrm{HF}$ is still lower than for some types of cancer, both in male and female populations (with the exception of lung cancer) [1]. Therefore, there is still a need for novel effective treatments for HF.

Recently, the results of studies evaluating the efficacy of sodium-glucose co-transporter 2 (SGLT2) inhibitors - dapagliflozin and empagliflozin - in the treatment of patients with HFrEF have been published [2]. SGLT-2 in-

\section{Corresponding author:}

Jadwiga Nessler, Department of Coronary Disease and Heart Failure, Institute of Cardiology, Jagiellonian University Medical College, Krakow, Poland, e-mail: jnessler@interia.pl

Received: 1.06.2021, accepted: 15.06.2021. 
hibitors are a new group of drugs that will undoubtedly mark another milestone in the therapy of patients with HFrEF. Dapagliflozin is, as yet, the only flozin approved for the treatment of patients with HFrEF. The DAPA-HF trial, which was the first RCT assessing the population of patients with $\mathrm{HF}$ with reduced ejection fraction with and without type 2 diabetes mellitus (T2DM), showed that dapagliflozin significantly reduced the risk of CV death and hospitalisation due to HF (26\%). This effect was equally pronounced in patients with T2DM and without concomitant carbohydrate metabolic disorders. In addition, a significant decrease in all-cause mortality in the DAPA-HF population was found in the subjects taking dapagliflozin compared to the placebo. In the dapagliflozin group, the patients had less severe $\mathrm{HF}$ symptoms than the subjects in the placebo group, which translated into a better quality of life, as evaluated by the Kansas City Cardiomyopathy Questionnaire (KCCQ) [2, 3].

The DAPA-HF trial is a ground-breaking study providing evidence for the efficacy of the novel drug group in the therapy of HF, and offering hope for further improvements in the prognosis of patients with HFrEF.

\section{Dapagliflozin - drug with beneficial mechanisms of action in heart failure with a reduced left ventricular ejection fraction}

Dapagliflozin has beneficial metabolic, nephroprotective and cardiovascular activity. These effects are due to the inhibitory action produced by dapagliflozin on SGLT2 receptors resulting in reduced reabsorption of glucose and sodium, which leads to their increased excretion with urine [4]. It also needs to be noted that reduced glucose reabsorption promotes a negative energy balance, causing weight loss and improving insulin sensitivity, and hence producing a favourable metabolic effect. The nephroprotective action of dapagliflozin is attributed to the contraction of the renal afferent arteriole, which reduces intraglomerular pressure and thus increases albumin excretion in urine. The $\mathrm{CV}$ effect comprises an improvement in haemodynamics and results from increased osmotic diuresis, reduced plasma volume, and decreased blood pressure, leading to lower left ventricular preload and afterload [5]. Dapagliflozin produces a diuretic action by inducing fluid loss from the intravascular compartment, which is highly beneficial in patients with HF, as it has no effect on reducing intravascular pressure, i.e. systolic ABP. Another key aspect is related to the positive impact of SGLT-2 inhibitors on myocardial energetics. SGLT2 inhibitors increase the production of ketone bodies and enhance their utilisation in the heart, which improves the energy metabolism of cardiomyocytes, and thus reduces the risk of HF development and recurrence [6]. Also, inhibition of the unfavourable process of cardiac remodelling has been observed [7].

\section{Indications for dapagliflozin treatment based on the drug's current SPC valid in Europe}

The results of the DAPA-HF trial, which were announced on 1 September 2019 at the European Congress of Cardiology in Paris and concurrently published in the New England Journal of Medicine, provide a basis for the use of dapagliflozin in a very broad population comprising not only patients with T2DM and HFrEF, but also patients with HFrEF without coexisting carbohydrate metabolic disorders. Aside from ARNIs, ACEIs (or ARBs), MRAs and $\beta$-blockers (BBs), dapagliflozin is as yet the only SGLT-2 inhibitor with proven efficacy in improving prognosis both in terms of all-cause and CV mortality in patients with HFrEF.

To summarise the data presented above, let me draw attention to some practical clinical considerations related to the use of dapagliflozin in patients with HFrEF.

On 15 October 2020, the Committee for Medicinal Products for Human Use (CHMP) adopted a positive opinion regarding dapagliflozin, extending its indications to include heart failure. In November 2020, dapagliflozin was approved in Europe - and hence in Poland - for the therapy of patients with $\mathrm{HFrEF}$ in the following indication: treatment of symptomatic chronic HFrEF in adults [8, 9].

On 30 April 2021, dapagliflozin was approved in the USA in the new indication of chronic kidney disease (CKD) in patients at risk of disease progression, both with and without T2DM. The drug's new indication was adopted on the basis of the phase III DAPA-CKD trial in which dapagliflozin showed an unprecedented reduction in the risk of a primary composite endpoint which included worsening of renal function, onset of end-stage renal disease, and cardiovascular (CV) or renal death [10].

\section{Status of dapagliflozin in the treatment of heart failure - target patient populations}

Based on the current Summary of Product Characteristics (SPC) valid in Europe, dapagliflozin is indicated for the treatment of symptomatic chronic HFrEF in adult patients in the following target groups [8]:

1. In patients with T2DM with or without coexisting HF, irrespective of the phenotype:

- in adult patients for the treatment of inadequately controlled T2DM, as an adjunct therapy to diet and exercise, either in monotherapy, in the absence of metformin tolerance, or in combination with other medicinal products used in the treatment of T2DM;

- in the treatment of inadequately controlled T1DM as an adjunct to insulin in patients with $\mathrm{BMI} \geq 27 \mathrm{~kg} / \mathrm{m}^{2}$, when insulin alone does not provide adequate glycaemic control despite optimal insulin therapy (dapagliflozin at a dose of $5 \mathrm{mg}$ ).

2. In adults for the treatment of symptomatic HFrEF

Dapagliflozin is the first SGLT-2 inhibitor officially approved for use in the population of adult HFrEF patients. 
Key clinical characteristics of patients who might benefit from dapagliflozin therapy:

- HFrEF with $\mathrm{EF} \leq 40 \%$,

- NYHA functional class II to IV,

- patients with or without T2DM,

- patients on standard cardiovascular treatment (i.e. $\beta$-blocker and/or renin-angiotensin-aldosterone system inhibitor (ACEI/ARB or ARNI) and MRA),

- dapagliflozin can be added to any HF therapy,

- creatinine clearance should be $\geq 30 \mathrm{ml} / \mathrm{min} / 1.73 \mathrm{~m}^{2}$ [2, 11].

Dapagliflozin therapy may be initiated either on discharge from hospital or on an outpatient basis. In patients with HFrEF, dapagliflozin is used at a dose of $10 \mathrm{mg}$ once daily in combination with other drugs indicated for the treatment of HF. No dose adjustment is necessary in patients with renal impairment, though it must be noted that the experience with using dapagliflozin in the treatment of HFrEF in patients with severe kidney dysfunction - similarly to the experience with dapagliflozin in patients with NYHA functional class IV - is fairly limited.

Kidney function should be assessed based on creatinine clearance according to the following schedule:

- before commencing treatment with dapagliflozin and at least once a year after that;

- before the initiation of drugs that may interfere with kidney function, and then periodically throughout treatment.

Dapagliflozin produces beneficial effects in patients taking medications recommended for the treatment of HFrEF. Dapagliflozin has been shown to act synergistically with drugs recommended for the therapy of HFrEF under applicable guidelines, regardless of the type of therapy and target doses used [11]. Similarly, the activity of dapagliflozin is unaffected by the doses of the primary pharmacotherapies and the application of implantable therapeutic devices (e.g. implantable cardioverter defibrillator (ICD) or cardiac resynchronisation therapy (CRT)). This observation implies an additional benefit associated with dapagliflozin regardless of optimised HFrEF pharmacotherapy or the application of therapeutic devices. The clinically relevant finding that the effect of dapagliflozin is independent of the patients' primary drug treatment upholds the hypothesis that SGLT2 inhibitors act in a mechanistically independent and complementary manner to other HFrEF therapies.

\section{Safety of dapagliflozin treatment}

Based on the published studies, dapagliflozin is a safe drug with a low reported incidence of adverse events (AEs) leading to treatment discontinuation. In view of the primary mechanism of action of SGLT2 inhibitors, it is not surprising that the most common AEs are fungal infections of the external urogenital tract. However, they tend to be mild to moderate in severity and, importantly, do not require discontinuation of SGLT2 inhibitor therapy. Infection symptoms resolve after treatment with a topical antifungal agent or a single dose of an antifungal drug. In the DAPA-HF trial, a total of $14(0.6 \%)$ patients in the dapagliflozin group, and $17(0.7 \%)$ patients in the placebo group, were found to experience serious events manifesting as urinary tract infections. Based on the available study findings, the risk of urinary tract infections in patients receiving SGLT2 inhibitor therapy is not elevated, and it should raise no concerns about the initiation of such treatment. It needs to be emphasised that a rare complication of therapy in patients with diabetes mellitus is ketoacidosis, especially in individuals with poor nutrition, with symptoms including polyuria, severe nausea and/or vomiting, abdominal pain, excessive thirst, respiratory distress, confusion, unusual fatigue or drowsiness. During dapagliflozin treatment particularly elderly patients may require adjustments of the dose of diuretics (especially loop diuretics). In diabetics, the initiation of dapagliflozin treatment may require adjustment of insulin and sulfonylurea therapy. It needs to be noted, though, that dapagliflozin does not increase the risk of hypoglycaemia $[11,12]$.

\section{Barriers to implementation of HF therapy}

The treatment of patients with HFrEF is based on RCT findings which are reflected in the guidelines issued by the European Society of Cardiology (ESC) or the European Heart Failure Association (HFA) [13, 14]. However, despite clearly defined guidelines and hard evidence confirming the efficacy of recommended therapies, their implementation and optimisation continue to be unsatisfactory [15]. Not all patients are prescribed medications according to the guidelines, despite appropriate indications for therapy, and a significant proportion take drugs at suboptimal doses. This may be due to problems arising from patients' intolerance to treatment used because of accompanying low ABP, renal impairment or hyperkalaemia [16-18]. It is fairly common for patients not to tolerate all therapies, at least at their target doses, so it may be necessary to determine which drugs will benefit the individual patient the most $[19,20]$. Other possible causes include difficulties with access to specialist care [21, 22], physician inertia or poor organisation of outpatient care [23].

Recently, the HFA has published a position paper outlining patient profiles that may be relevant for the implementation of HFrEF treatment. The paper stresses the need for identifying the causes of undertreatment and, secondly, implementing appropriate treatment in patients with HF. The causes of inadequate treatment can be related to "non-medical" factors such as poor socioeconomic status, lack of social support, and non-adherence. However, they are frequently associated with medical conditions such as low ABP, renal dysfunction or persistent congestion. The authors of the position 
paper highlight that the immediate period after hospital discharge is very important, as it carries a very high risk of another exacerbation. There is evidence that HFrEF patients receiving guideline-directed medical therapy (GDMT) have a better prognosis at discharge [24].

Recent reports have shown that ARNI and SGLT2 inhibitors can be safely introduced into treatment regimens before patient discharge from hospital, which helps to achieve a reduction in hospitalisation and mortality rates [25-27]. Also, there is solid evidence that patient adherence is associated with a lower risk of CV complications and mortality, and fewer HF-related hospitalisations $[28,29]$.

\section{Patient phenotyping for targeted therapies}

With novel effective therapies for HF, phenotyping is increasingly required, as some patients cannot tolerate all medications. Patients with HF pose a challenge, as the causal phenotypes overlap with the clinical phenotypes of HF. Therefore, selecting appropriate therapy requires patient stratification combining clinical characteristics, biomarker levels, and various imaging techniques. Patients with HF have many different presentations related to congestion, haemodynamic status, and kidney function. Consequently, adjusting treatment regimens to patient profiles seems a reasonable way to ensure that every patient benefits from GDMT. The mainstays of HF treatment are ACEIs/ARBs/ARNIs, BBs, MRAs and - since recently-SGLT2 inhibitors. According to the current state of knowledge, these drugs should be the starting point for treatment of the majority of patients with HF. All the drug groups listed above, except for SGLT2 inhibitors (which have only a minor effect on $A B P$ ), significantly lower systolic $A B P$ and affect serum potassium levels, requiring dose adjustment and gradual up-titration. Therefore, SGLT2 inhibitors can be safely incorporated into combination HF therapy.

The authors of the consensus document identify nine phenotypes of patients with individual needs for dose up-titration [24]:

1. Patients with low blood pressure and high heart rate

In patients with symptomatic hypotension, unnecessary ABP lowering medications must be eliminated. In such cases, ivabradine, whose sole effect is on reducing heart rate (HR), with no impact on $A B P$, can be used. MRAs and SGLT2 are also known to have a small impact on ABP. 2. Patients with low blood pressure and low heart rate

Modification of GDMTs or their doses is necessary only in patients with symptomatic hypotension. MRAs and SGLT2 inhibitors have little effect on ABP. A dose reduction of BBs may be necessary if the patient has a resting heart rate $<50 \mathrm{bpm}$, or symptomatic bradycardia.

3. Patients with normal blood pressure and low heart rate

If possible, drugs with a negative chronotropic effect should be discontinued, including non-dihydropyridine calcium channel blockers (diltiazem and verapamil), di- goxin, or antiarrhythmic drugs. In patients taking ivabradine, the dose should be reduced or the drug should be discontinued if the HR persists at a level $<50 \mathrm{bpm}$ or the patient has symptomatic bradycardia. In addition, patients with bradycardia or $\mathrm{HR}<50 \mathrm{bpm}$ require a reduction of BB dose.

4. Patients with normal blood pressure and high heart rate

These patients should be treated with the target doses of BBs. If high heart rate in sinus rhythm (> $70 \mathrm{bpm}$ ) persists, BBs in combination with ivabradine should be used. In hospitalised patients, the initiation of vericiguat should be considered before discharge.

5. Patients with atrial fibrillation and normal blood pressure

There is no clear evidence for any prognostic benefits of $\mathrm{BBs}$ in patients with $\mathrm{HF}$ and $\mathrm{AF}[30,31]$. Attempts to optimise the dosage of BBs up to the maximum tolerated dose may have a detrimental effect, as ventricular rates $<70$ bpm have been found to be associated with a worse outcome.

6. Patients with atrial fibrillation and low blood pressure

Digoxin may be used as an alternative to BBs. MRAs and SGLT2 inhibitors have very little effect on $A B P$, so they can be used in this patient group.

7. Patients with chronic kidney disease

ACEIs/ARBs/ARNIs should only be withdrawn if creatinine increases by $>100 \%$ or to $>3.5 \mathrm{mg} / \mathrm{dl}$, or the estimated glomerular filtration rate (eGFR) decreases to $<20 \mathrm{ml} / \mathrm{min} / 1.73 \mathrm{~m}^{2}$. BBs can be safely used in patients when their eGFR is not lower than $30 \mathrm{ml} / \mathrm{min} / 1.73 \mathrm{~m}^{2}$. MRAs can also be used if the eGFR is $>30 \mathrm{ml} / \mathrm{min} / 1.73 \mathrm{~m}^{2}$, and the potassium level is $\leq 5.0 \mathrm{mmol} / \mathrm{l}$. Blood testing for potassium levels should be performed at 1 and 4 weeks after starting MRA treatment or increasing the dose, and then periodically during follow-up visits. ARNIs can be used up to the eGFR values of $30 \mathrm{ml} / \mathrm{min} / 1.73 \mathrm{~m}^{2}$. Empagliflozin has been shown to be effective and safe, and improve renal endpoints, in patients with an eGFR $>20-25 \mathrm{ml} / \mathrm{min} / 1.73 \mathrm{~m}^{2}$. Also, there is recent evidence of benefits associated with using dapagliflozin (DAPA-CKD) also in patients with eGFR $<20 \mathrm{ml} / \mathrm{min} / 1.73 \mathrm{~m}^{2}$ [11].

8. Pre-discharge patient

In patients who are not on BB treatment, drugs from this group should not be introduced as first-line therapy, as they may lead to clinical deterioration in congestive patients. However, ACEls or ARNIs can be initiated, provided that patients' systolic blood pressure is $>90$ or $>100 \mathrm{~mm} \mathrm{Hg}$ [25]. MRAs and SGLT2 inhibitors can be introduced safely even in patients with low ABP.

9. Patient with hypertension despite guideline-directed medical therapy

In patients with arterial hypertension, it is important to ensure that they do not take any medications that may cause ABP elevation (such as non-steroidal anti-inflammatory drugs, corticosteroids or bronchodilators). 


\section{Conclusions}

An analysis of individual patient phenotypes shows that dapagliflozin is a drug that stands a chance of being used in the majority of patients with HF. Based on the beneficial metabolic, renal and haemodynamic profiles, dapagliflozin is suitable for use in groups of patients with low $A B P, C K D$, and congestion. In view of the high clinical efficacy of dapagliflozin (reduction in mortality, CV mortality and HF-related hospitalisation rates) combined with an early onset of clinical effects (observed already approximately 28 days after the initiation of treatment), a significant improvement in the quality of life, high safety of the therapy and easy dosing (without the need for titration), treatment with this drug should be considered as early as possible after the diagnosis of HF in most patients. This is particularly relevant nowadays, in the time of the COVID-19 pandemic, when patients with HF need a therapy that is simple to dose, does not require frequent follow-ups, and produces the desired therapeutic effects quickly.

The use of therapies recommended by appropriate guidelines plays a crucial contributory role in reducing the mortality and morbidity in patients with HF. Consequently, every effort should be made to initiate and then optimise primary therapy. A personalised approach to patients - taking into account their individual haemodynamic profiles (ABP, HR, presence of congestion) and renal function - may result in improved achievement of therapeutic goals compared to the traditional "step-bystep" treatment requiring the target doses of each drug group to be reached before adding another. It is important to be aware that the "one size fits all" approach has not worked for many HF patients.

\section{Conflict of interest}

The author declares no conflict of interest.

\section{References}

1. Mamas MA, Sperrin M, Watson MC, et al. Do patients have worse outcomes in heart failure than in cancer? A primary carebased cohort study with 10-year follow-up in Scotland. Eur J Heart Fail 2017; 19: 1095-104.

2. McMurray JJV, Solomon SD, Inzucchi SE, et al.; DAPA-HF Trial Committees and Investigators. Langkil de AM. Dapagliflozin in patients with heart failure and reduced ejection fraction. N Engl J Med 2019; 381: 1995-2008.

3. Packer M, Anker SD, Butler J, et al.; EMPEROR-Reduced Trial Investigators. Cardiovascular and renal outcomes with empagliflozin in heart failure. N Engl J Med 2020; 383: 1413-24.

4. Verma S, MCMurray JJV. SGLT2 inhibitors and mechanisms of cardiovascular benefit: a state-of-the-art review. Diabetologia 2018; 61: 2108-17.

5. Heerspink HJL, Perkins BA, Fitchett DH, et al. Sodium glucose cotransporter 2 inhibitors in the treatment of diabetes mellitus: cardiovascular and kidney effects, potential mechanisms, and clinical applications. Circulation 2016; 134: 752-72.
6. Heerspink HJL, Kosiborod M, Inzucchi SE, Cherney DZI. Renoprotective effects of sodium-glucose cotransporter-2 inhibitors. Kidney Int 2018; 94: 26-39.

7. Tamargo J. Sodium-glucose cotransporter 2 inhibitors in heart failure: potential mechanisms of action, adverse effects and future developments. Eur Cardiol 2019; 14: 23-32.

8. Charakterystyka produktu leczniczego FORXIGA 10 mg. 2020.

9. European Medicines Agency; Forxiga dapagliflozin. 2020; 1: 1-2. https:// www.ema.europa.eu/en/medicines/human/summaries-opinion/forxiga-1 (9.11.2020).

10. Nessler J, Siniarski A, Leszek P, et al. Expert opinion of the Heart Failure Working Group of the Polish Cardiac Society on the use of dapagliflozin in the treatment of heart failure with reduced ejection fraction. Kardiol Pol 2021; 79: 363-71.

11. Heerspink HJL, Stefánsson BV, Correa-Rotter R, et al.; DAPA-CKD Trial Committees and Investigators. Dapagliflozin in patients with chronic kidney disease. N Engl J Med 2020; 383: 1436-46.

12. McMurray JJV, Solomon SD, Inzucchi SE, et al.; DAPA-HFTrial Committees and Investigators. Langkilde AM. Dapagliflozin in patients with heart failure and reduced ejection fraction. N Engl J Med 2019; 381: 1995-2008.

13. Seferovic PM, Ponikowski P, Anker SD, et al. Aktualizacja praktyki klinicznej o niewydolności serca 2019: farmakoterapia, procedury, urządzenia i postępowanie z pacjentem. Spotkanie konsensualne ekspertów raport Towarzystwa Niewydolności Serca Europejskiego Towarzystwa Kardiologicznego. Eur J Heart Fail 2019; 21: 1169-86.

14. Seferović PM, Coats AJS, Ponikowski P, et al. Europejskie Stanowisko Society of Cardiology/Heart Failure Association dotyczące roli i bezpieczeństwa nowych leków obniżających stężenie glukozy u pacjentów z niewydolnością serca. Eur J Heart Fail 2020; 22: $196-213$.

15. Thorvaldsen T, Benson L, Dahlström U, et al. Use of evidence-based therapy and survival in heart failure in Sweden 2003-2012. Eur J Heart Fail 2016; 18: 503-11.

16. Crespo-Leiro MG, Barge-Caballero E, Segovia-Cubero J, et al. Hyperkalemia in heart failure patients in Spain and its impact on guidelines and recommendations: ESC-EORP-HFA Heart Failure Long-Term Registry. Rev Esp Cardiol 2020; 73: 313-23.

17. Trevisan M, de Deco P, Xu H, et al. Incidence, predictors and clinical management of hyperkalaemia in new users of mineralocorticoid receptor antagonists. Eur J Heart Fail 2018; 20: 1217-26. Erratum in: Eur J Heart Fail 2019; 21: 540.

18. Rossignol P, Lainscak M, Crespo-Leiro MG, et al.; Heart Failure Long-Term Registry Investigators Group. Unravelling the interplay between hyperkalaemia, renin-angiotensin-aldosterone inhibitor use and clinical outcomes. Data from 9222 chronic heart failure patients of the ESC-HFA-EORP Heart Failure Long-Term Registry. Eur J Heart Fail 2020; 22: 1378-89.

19. Chioncel O, Lainscak M, Seferovic PM, et al. Epidemiology and one-year outcomes in patients with chronic heart failure and preserved, mid-range and reduced ejection fraction: an analysis of the ESC Heart Failure Long-Term Registry. Eur J Heart Fail 2017; 19: 1574-85.

20. Packer M, Metra M. Guideline-directed medical therapy for heart failure does not exist: a non-judgmental framework for describing the level of adherence to evidence-based drug treatments for patients with a reduced ejection fraction. Eur J Heart Fail 2020; 22: 1759-67.

21. Lund LH, Braunschweig F, Benson L, et al. Association between demographic, organizational, clinical, and socio-economic char- 
acteristics and underutilization of cardiac resynchronization therapy: results from the Swedish Heart Failure Registry. Eur J Heart Fail 2017; 19: 1270-9.

22. Savarese G, Carrero JJ, Pitt B, et al. Factors associated with underuse of mineralocorticoid receptor antagonists in heart failure with reduced ejection fraction: an analysis of 11215 patients from the Swedish Heart Failure Registry. Eur J Heart Fail 2018; 20: 1326-34.

23. Verhestraeten C, Heggermont WA, Maris M. Clinical inertia in the treatment of heart failure: a major issue to tackle. Heart Fail Rev 2020 May 30. doi: 10.1007/s10741-020-09979-z.

24. Rosano GMC, Moura B, Metra M, et al. Patients profiling in heart failure for tailoring medical therapy. A consensus document of the Heart Failure Association of the European Society of Cardiology. Eur J Heart Fail 2021 DOI: 10.1002/ejhf.2206.

25. Wachter R, Senni M, Belohlavek J, et al.; TRANSITION Investigators. Initiation of sacubitril/valsartan in haemodynamically stabilised heart failure patients in hospital or early after discharge: primary results of the randomised TRANSITION study. Eur J Heart Fail 2019; 21: 998-1007.

26. Damman K, Beusekamp JC, Boorsma EM, et al. Randomized, double-blind, placebo-controlled, multicentre pilot study on the effects of empagliflozin on clinical outcomes in patients with acute decompensated heart failure (EMPA-RESPONSE-AHF). Eur J Heart Fail 2020; 22: 713-22.

27. Bhatt DL, Szarek M, Steg PG, et al.; SOLOISTWHF Trial Investigators. Sotagliflozin in patients with diabetes and recent worsening heart failure. N Engl J Med 2021; 384: 117-28.

28. Calvin JE, Shanbhag S, Avery E, et al. Adherence to evidencebased guidelines for heart failure in physicians and their patients: lessons from the Heart Failure Adherence Retention Trial (HART). Congest Heart Fail 2012; 18: 73-8.

29. Komajda M, Schöpe J, Wagenpfeil S, et al.; QUALIFY Investigators. Physicians' guideline adherence is associated with longterm heart failure mortality in outpatients with heart failure with reduced ejection fraction: the QUALIFY international registry. Eur J Heart Fail 2019; 21: 921-9.

30. Kotecha D, Holmes J, Krum H, et al. Efficacy of $\beta$ blockers in patients with heart failure plus atrial fibrillation: an individual-patient data meta-analysis. Lancet 2014; 384: 2235-43.

31. Cleland JGF, Bunting KV, Flather MD, et al. Beta-blockers in Heart Failure Collaborative Group. Beta-blockers for heart failure with reduced, mid-range, and preserved ejection fraction: an individual patient-level analysis of double-blind randomized trials. Eur Heart J 2018; 39: 26-35. 